\title{
Diversité et coexistence des approches pédagogiques
}

Diversity and multiplicity in pedagogical approaches - the Italian experience

Diversidad y coexistencia de los enfoques pedagógicos. El caso italiano

\section{Teresa Longo}

\section{OpenEdition}

\section{Journals}

Édition électronique

URL : https://journals.openedition.org/ries/1884

DOI : $10.4000 /$ ries. 1884

ISSN : 2261-4265

Éditeur

France Education international

Édition imprimée

Date de publication : 1 avril 2002

Pagination : $91-100$

ISBN : 2-84520-553-8

ISSN : $1254-4590$

\section{Référence électronique}

Teresa Longo, « Diversité et coexistence des approches pédagogiques », Revue internationale d'éducation de Sèvres [En ligne], 29 | avril 2002, mis en ligne le 24 novembre 2011, consulté le 07 juillet 2021. URL : http://journals.openedition.org/ries/1884 ; DOI : https://doi.org/10.4000/ries.1884

\section{(c) Tous droits réservés}




\section{Diversité et coexistence des approches pédagogiques}

\section{Le cas de l'Italie}

\section{Teresa Mariano Longo}

S’il est vrai que les idées pédagogiques en vigueur à une époque et la philosophie qu'elles sous-tendent constituent des bases importantes de l'organisation et des contenus du travail de l'élève, la culture qui inspire l'organisation du temps de l'école dans la vie d'un jeune est en fait encore plus importante: temps de l'étude et temps de "la vie» des enfants peuvent être conçus comme deux moments qui s'opposent ou s'harmonisent selon le sens que l'école et le contexte social donnent à l'instruction et donc à un projet de vie.

C'est ainsi que tout voyageur français qui visite les écoles d'Italie reste étonné par les différences qu'il observe dans l'organisation du temps d'école : le temps d'école en Italie s'étale tout au long de dix mois de l'année sans interruption, sauf pour Noël et si, au quotidien, le temps d'école est plus court pour un jeune Italien que pour un Français (il entre en classe à $8 \mathrm{~h}$ et en sort à $13 \mathrm{~h}$ ), la semaine est de six jours. La culture française sépare le temps du travail du temps de la "récréation », en Italie les deux temps s'entremêlent. Comment à l'intérieur de cette grande différence culturelle le travail de l'élève italien est-il organisé ?

L'analyse des prescriptions concernant le curriculum peut contribuer à nous faire comprendre les choix d'un pays, à un moment historique particulier, en matière d'éducation et d'organisation du temps de l'enfant ; toutefois, c'est surtout l'étude des pratiques, du «curriculum réel », qui peut nous montrer comment le travail des enfants et des jeunes se définit.

En Italie, les prescriptions du gouvernement central en matière de travail scolaire sont rares : les établissements et les enseignants choisissent librement les activités des élèves, mais en tenant compte des parents et de leurs stratégies d'emploi du temps.

Dans cet article, je chercherai à présenter les modalités les plus diffusées de l'organisation du travail de l'élève en Italie et les questions en débat sur ce sujet. Après avoir fourni au lecteur étranger à la réalité italienne quelques rapides références historiques, je présenterai ensuite ce qu'il en est des "prescriptions institutionnelles », avant d'analyser trois modalités du travail scolaire "réel », en regard des différentes approches pédagogiques qui leur correspondent. 


\section{L'HISTOIRE}

L'école élémentaire publique italienne trouve ses origines dans les écoles communales. Jusqu'en 1911, en effet, les communes avaient compétence sur la durée de la scolarité et sur le calendrier scolaire. Peu d'enfants fréquentaient l'école à la fin du $\mathrm{XIX}^{\mathrm{e}}$ siècle et les premières lois du royaume d'Italie sur l'obligation scolaire imposaient l'instruction pour le premier cycle élémentaire, mais non la scolarisation (Loi Coppino 1877). L'obligation scolaire était une obligation de connaître un programme pour se présenter à un examen, mais la préparation de ce dernier pouvait se faire dans des lieux autres que l'établissement scolaire. Les écoles étaient d'ailleurs rares et peu d'enfants les fréquentaient : les enfants des familles aisées se préparaient chez eux avec un précepteur ; les enfants des familles pauvres ou des zones éloignés de l'école, quant à eux, s'ils voulaient se préparer à l'examen, pouvaient le faire en se procurant un manuel. Le programme d'examen et le manuel étaient les références principales pour accomplir l'obligation scolaire.

Ce n'est qu'au début du $\mathrm{XX}^{\mathrm{e}}$ siècle que s'affirme l'idée d'une école publique présente sur tout le territoire national et prenant en charge «l'éducation " de l'enfant. À l'époque, deux philosophies s'opposent en proposant deux conceptions différentes du temps et du travail de l'enfant: le scientisme, qui donne une importance majeure à l'« l'hygiène de vie » et aux rythmes biologiques de l'enfant et l'idéalisme qui donne plus d'importance à la formation de l'« esprit » et qui considère que c'est le programme, et non le corps de l'enfant, qui doit scander les rythmes scolaires.

La réforme du système scolaire de Gentile (1923) s'inspire de cette dernière philosophie : le régime fasciste " $s$ 'approprie du temps de l'enfant ${ }_{\text {.... }}$ "il faut travailler, étudier et surtout obéir» (Ragazzini, 1997). La pédagogie s'organise dans une institution hiérarchiquement ordonnée : les programmes, le manuel, le directeur, l'inspecteur, le professeur, les devoirs, les notes. La culture de l'école est alors surtout orale : l'élève doit réciter et répéter ce que le maître a dit. La rhétorique, le discours constituent en effet des valeurs du régime. Il ne faut à cet égard d'ailleurs pas oublier que le discours oral sert aussi à contrôler et à corriger les façons de prononcer et de parler des enfants d'une nation ayant de nombreux dialectes et langues.

L'école de l'après-guerre change lentement au plan institutionnel, mais c'est immédiatement que les enseignants se mettent à exercer leur "liberté didactique » et que la culture fasciste est soumise à la critique. Un point, toutefois : l'oral continue de dominer.

En 1963 une importante réforme modifie en profondeur l'école obligatoire : l'école moyenne unifiée (Scuola Media) est créée, d'une durée de trois ans, et l'obligation scolaire est reportée à l'âge de quatorze ans. Le cadre de la démocratisation de l'accès aux études est ainsi tracé, mais d'importantes contradictions se font aussitôt jour car la manière d'enseigner et d'organiser le travail 
de l'élève est restée la même qu'auparavant, toujours fondée sur le cours magistral, le manuel et l'interrogation orale.

On remarque en particulier que l'organisation du temps et de la vie de l'enfant est déterminante pour la réussite scolaire, comme l'écrit Don Milani en 1967: « la vieille école était faite pour les classes aisées d'abord par ses horaires et son calendrier. La nouvelle école ne les a pas changés. Elle reste une culture pour les riches... mais il y a une espérance : l'école à temps plein » (p. 31). C'est dans ce contexte en effet que les ragazzi di Barbiana défendent l'idée qu'une journée de travail scolaire plus longue peut contribuer à compenser les écarts culturels d'origine entre les enfants. L'opinion conservatrice italienne est alors d'avis opposé et l'école à temps plein a eu du mal à s'imposer : le débat dura une décennie et ce sont finalement les politiques d'austérité qui en eurent raison.

Dans l'intervalle, toutefois, les enseignants et les organisations de la société civile réussirent à mettre en place des "écoles à temps plein » et à conduire des expérimentations ayant comme objectifs la lutte contre l'échec scolaire et la construction d'une culture opposée à la vieille culture orale. Un décret de 1983 sur la Scuola Media et la loi de 1990 qui réforme l'école primaire reconnaissent le temps prolongé ou «temps plein ». Malgré cette évolution juridique, toutefois, il est à noter que les politiques de restrictions budgétaires et la remise en cause du service public d'éducation ont rendu difficile la mise en œuvre de cette réforme sur tout le territoire national.

\section{LA SITUATION ACTUELLE : LES NORMES ET LES PRATIQUES}

\section{Les prescriptions}

Les programmes officiels relatifs aux trois niveaux d'école ${ }^{1}$ ne donnent aucune indication sur les méthodes didactiques et sur le travail des élèves. Il n'existe pas non plus, comme c'est le cas en France et dans d'autres pays, de corps d'inspecteurs chargés de contrôler la bonne application des normes, le choix des méthodes et des manières d'enseigner. L'enseignant, en Italie, en revanche, quand il organise le travail de l'élève, doit tenir en compte des

\footnotetext{
1. Structure de l'école italienne : à l'âge de six ans les enfants entrent à l'école élémentaire pour 5 ans; à onze ans ils entrent à l'école Media qui dure trois ans et qui se termine par un examen. Jusqu'à 14 ans les programmes scolaires sont les mêmes pour tous ; après la Media les élèves entrent à l'école secondaire et ont à choisir entre le Liceo (classique, scientifique, pédagogique, artistique), les instituts techniques et les instituts professionnels. L'école obligatoire se termine à 15 ans, après une année d'école secondaire.

Ceux qui veulent poursuivre leurs études secondaires supérieures le font pendant un triennium à la fin duquel ils présentent l'examen de maturità. La structure est donc de $5+3+(2+3)=13$ ans. En 1999 une réforme qui élevait l'âge de l'obligation scolaire de 14 à 15 ans, avait ramené à 12 ans la durée de la scolarité et organisé l'école obligatoire selon une structure en cycles, éliminant du même coup la Media. Le gouvernement actuel a suspendu l'application de cette réforme qui aurait dû démarrer en 2001 et proposait une structure de $5+3+4$.
} 
décisions des conseils de gestion de l'école et des normes officielles relatives à l'évaluation ${ }^{2}$.

Depuis les années soixante-dix, en effet, la liberté didactique des enseignants s'est trouvée confrontée à la présence des parents, des élèves et de diverses composantes de la société au sein des conseils de gestion des établissements : en 1974, une importante réforme introduit dans les établissements de nouvelles instances de gestion en même temps qu'elle attribue à chaque établissement plus d'autonomie pour l'expérimentation didactique, via le « conseil des enseignants », qui a un pouvoir décisionnel sur les projets pédagogiques. Au sein du « conseil de classe », par ailleurs, les parents expriment leur avis sur le déroulement de la vie scolaire et leurs attentes vis-à-vis de l'école : à noter que le travail des élèves est un thème qui est toujours à l'ordre du jour de ces conseils, où la famille confronte son idée du temps à celle de l'école. En conséquence, quand un enseignant décide de recourir à telle ou telle méthode, il doit tenir compte des points d'accord qui ont été obtenus dans toutes ces instances, mais aussi des décisions du conseil des enseignants, de celles prises collégialement par ses collègues de la même discipline, ainsi que de l'avis du proviseur, qui exerce un rôle de plus en plus actif dans le domaine pédagogique.

Récemment (1997-1999), la loi a donné plus d'autonomie aux établissements : chaque école élabore un plan d'offre de formation (POF) pour indiquer son projet de formation, à partir de son environnement, de ses élèves et des ressources disponibles. Les POF s'attachent rarement aux méthodes didactiques et, quand c'est le cas, le font de manière peu approfondie.

L'établissement doit respecter la dotation horaire globale pour l'année et le cycle prévue par les programmes nationaux : $24 \mathrm{~h}$ pour le primaire, $30 \mathrm{~h}$ pour la Media, autour de $28 \mathrm{~h}$ pour l'école secondaire. Il est libre de gérer cette dotation selon les critères pédagogiques qu'il considère les plus opportuns et définit donc en début d'année son calendrier de l'année (en accord avec l'autorité locale), l'horaire hebdomadaire et la répartition des heures entre les disciplines (non le nombre des disciplines). À l'exception des écoles « à temps plein " ${ }^{3}$, l'école occupe essentiellement la matinée. À noter au passage que cela ne signifie pas que les Italiens fréquentent l'école pour un temps inférieur à leurs collègues européens ${ }^{4}$.

La loi sur l'Autonomie prévoit que les programmes sont divisés en une partie nationale et une partie locale ${ }^{5}$. Si la tradition de liberté didactique de chaque enseignant est toujours importante, le proviseur a désormais un droit de

2. Un enseignant n'est inspecté, en Italie, que dans le cas où le proviseur juge négativement son travail et où il en fait la demande au ministère. Ces interventions sont rares.

3. À l'école primaire $21 \%$ des classes fonctionnent à temps plein ; à l'école Media les classes à temps plein sont rares et $30 \%$ des classes fonctionnent « à temps prolongé » (Ministero dell'Istruzione, 2001)

4. Par exemple : à l'âge de sept ans un enfant français va à l'école 846 heures par an, un enfant italien 900 heures. À l'école secondaire inférieure en France (collège) les élèves fréquentent l'école pour un minimum de $841 \mathrm{~h}$ et un maximum de $990 \mathrm{~h}$ par an, la fourchette italienne étant entre $933 \mathrm{~h}$ et $1260 \mathrm{~h}$. (Eurydice 1999. Données 1997/98) 5. Pour en savoir plus sur l'autonomie des établissements en Italie, voir Angela Martini : « Le plan d'offre de formation entre illusion et réalité » in Politiques d'éducation et de formation, 2001/1 De Boeck Université, Bruxelles. 
regard : il peut inspecter les cours et, en accord avec le conseil des enseignants et le conseil de classe, il décide des critères d'évaluation et du nombre de contrôles écrits et oraux. À ce sujet, le ministère de l'éducation s'est prononcé une seule fois en recommandant que chaque enseignant évalue ses élèves à partir d'un nombre "convenable" d'épreuves (orales et écrites selon la discipline). À la fin de chaque quadrimestre, la famille reçoit un carnet sur lequel, si l'enfant fréquente l'école obligatoire, figure une évaluation des résultats sur la base de 8 valeurs (d' " excellent " jusqu'à " très insuffisant ») et un bref compte rendu des progrès de l'élève dans chaque discipline. À la fin de chaque bimestre, l'école doit par ailleurs informer les familles des résultats partiels.

Dans l'école secondaire supérieure a été institué récemment un système dit des « lacunes à combler » qui entend responsabiliser les élèves pour qu'ils entreprennent de combler leur lacunes ${ }^{6}$. Contre l'échec scolaire, les écoles doivent prévoir elles-mêmes le soutien scolaire ou le rattrapage : il s'agit dans les établissements soit d'activités pour la classe entière (p.e. explicitation des méthodes et des objectifs), de pauses à finalité didactique dans le traitement du programme, d'activités par " groupes de niveau » ou encore de la pratique dite du " guichet », qui a connu une récente fortune : les élèves s'adressent individuellement, en dehors des heures de cours, à leur professeur soit pour leur demander des explications sur des points de programme, soit pour des problèmes personnels relatifs à leur scolarité.

Enfin, pour terminer sur les aspects prescriptifs, je voudrais mettre en évidence le fait que, au cours de ces quarante dernières années, le pouvoir politique n'est intervenu directement sur le travail des élèves qu'en deux occasions : en 1969, pour que la fête du Dimanche soit respectée et donc pour interdire le contrôles et les interrogations du lundi et en 1998, par un décret du président de la République concernant le "statut des élèves ", qui formalise les termes du contrat entre l'école et l'élève et affirme notamment, dans un article, le devoir des élèves de respecter leurs engagements à étudier. Une disposition donc très générale du pouvoir central dans un contexte où l'établissement et l'enseignant, dotés d'une grande autonomie, décident du travail de l'élève.

\section{LES PRATIQUES : APPROCHES PÉdAgogiques ET TRAVAIL DE L’ÉLÈVE}

Si l'on compare la situation de l'enseignement italien à celle d'autres pays, à celle de la France, par exemple, les pratiques des enseignants sont, de toute évidence, en Italie, très différenciées. Ce serait toutefois une erreur de conclure à

6. Il s'agit d'un système adopté au moment de la suppression de l'examen de septembre. Auparavant, l'élève qui n'obtenait pas la moyenne pouvait, pour passer au niveau supérieur, passer un examen en septembre dans trois disciplines maximum. La réforme de 1996 substitue à ce système celui des " dettes de formation " : si l'élève ne comble pas les lacunes constatées au cours des dernières années d'école secondaire, on soustraira des points en conséquence à la note évaluant les résultats de son travail scolaire lors de l'examen final (maturità). 
une situation d'éparpillement : si l'on regarde en effet plus attentivement la diversité des situations italiennes, il est possible de voir émerger une typologie de pratiques, constituées à partir des valeurs éducatives présentes dans la société civile, des dynamiques entre enseignants et familles et des idées pédagogiques qui ont la faveur des enseignants. La « mise au travail » des enfants change selon l'approche que l'enseignant choisit. Pour ce qui concerne l'école obligatoire trois types d'approches semblent émerger.

\section{L'approche traditionnelle}

Cette approche, qui poursuit la tradition orale ${ }^{7}$ de l'école italienne, est surtout développée à l'école primaire et Media à temps court (très rarement dans celle à temps plein). À l'école primaire, l'enseignant récite plus ou moins mécaniquement son cours, sur la base du manuel et en s'aidant du tableau noir : l'élève, quant à lui, écoute. Le cahier d'exercices et le manuel sont les outils du travail de l'enfant. La tenue et l'ordre du cahier sont jugés très importants et souvent pris en compte pour l'évaluation. Le dessin est la seule activité qui se distingue en faisant appel à la créativité de l'enfant. Même si l' «éducation au mouvement » fait partie des programmes, l'enseignant traditionnel l'évite : cette question est bien sûr liée au problème du rapport entre vie à l'intérieur et à l'extérieur de l'école, puisque si les enfants ne fréquentent l'école que le matin, les activités physiques sont laissées à l'initiative des familles. La plupart des familles inscrivent les enfants à des cours particuliers de football, tennis, natation, hors de l'école.

À l'école Media l'importance donnée à l'oral est caractéristique de la conception qui a cours de ce niveau d'instruction. Comme ailleurs, en effet, l'école secondaire inférieure a un rôle ambigu : poursuite de l'école primaire? Initiation aux études du « secondaire supérieur » ? En général l'enseignant « traditionnel » privilégie cette approche. L'enseignant récite son cours afin de « faire passer » une certaine partie du programme de la discipline et certaines notions : le cours magistral occupe la plus grande part du temps scolaire, le reste étant consacré à l'interrogation orale.

À la Media, en italien et mathématiques, les contrôles doivent être aussi écrits. Pour l'italien il s'agit de résumés, d'analyses de texte et de rédactions (le fameux tema).

Dans toutes les autres disciplines l'évaluation a comme base l'oral. L'enseignant traditionnel donne une importance majeure aux devoirs à la maison, qui, dans les dernières classes de l'école primaire et à la Media ne se

\footnotetext{
7. L'importance de l'oral dans le système d'instruction italien a son origine dans l'interprétation idéaliste de la culture humaniste : un esprit savant s'exprime nécessairement par un bon discours. La parole étant le reflet de l'esprit, l'école idéaliste éduque surtout à la rhétorique sans porter attention aux faits eux-mêmes, à l'expérimentation scientifique et à la construction des connaissances.
} 
limitent pas à des exercices sur le cours tel qu'il a été enseigné, mais sont des vrais compléments aux programmes, censés les développer.

Le devoir à la maison pour les enfants de l'école primaire à temps partiel consiste en quelque exercice qui ne lui prend pas trop de temps dans les premières classes. Toutefois, plus on avance dans la scolarité, plus les enseignants considèrent qu'il est nécessaire de travailler à la maison pendant un certain nombre d'heures et plus ils prescrivent de devoirs. À douze ans, les devoirs occupent au moins trois heures de la journée d'un jeune.

À la Media, l'enseignant fait une distinction entre les devoirs pour «le jour d'après » (pour lesquels il faut seulement réviser ce qui a été dit en classe) et les devoirs bénéficiant d'un délai plus long, ce qui demande à l'enfant de planifier ses activités : il s'agit de la lecture et du résumé d'un livre, d'une dissertation, en quelques cas, ou encore d'une petite recherche. La participation d'un membre de la famille à ce type de devoir est très importante.

En classe, l'enseignant contrôle les cahiers d'exercice, se livre à de courtes interrogations et vérifie si l'élève a étudié chez lui. Deux ou trois fois par quadrimestre, il soumet l'élève à de longues interrogations orales (vingt minutes à l'estrade) : à cette occasion, l'élève doit savoir traiter un sujet et répondre aux questions qu'il lui pose. Ce système d'interrogation a cours aussi à l'école secondaire supérieure, l'interrogation "longue» ayant un poids important dans le jugement final. Ce n'est toutefois pas la somme des interrogations qui détermine l'appréciation finale : pour chaque matière, en effet, la note d'un quadrimestre est le résultat à la fois des notes obtenues aux épreuves, de l'avis de l'enseignant de la discipline et de celui du conseil des enseignants de la classe, qui porte sur le travail et le comportement de l'élève. À la Media, l'éducation physique fait partie des disciplines prévues aux programmes, même si les activités sportives et artistiques sont en général prises en charge par les familles hors de l'école.

Au niveau de la Media toujours, la bonne exécution des devoirs à la maison est nécessaire à la réussite scolaire, le rôle de la famille pour éduquer l'enfant à gérer son temps personnel étant capital. La capacité/possibilité des parents à aider l'enfant à trouver un équilibre entre travail scolaire et temps libre détermine la bonne réussite scolaire. L'approche traditionnelle, dans l'école à temps court, continue à poser les problèmes dénoncés par Don Milani, en ce qu'elle renforce les différenciations sociales.

Il n'en est pas moins vrai que de nos jours les comportements des familles semblent refléter l'évolution des mentalités dans la société italienne : l'idée a fait son chemin selon laquelle, après l'école, l'enfant doit "faire sa vie d'enfant ", ce qui veut dire jouer, bouger, voir ses copains et se reposer. Cette idée, toutefois, même très répandue, est mise en pratique de différentes manières : la famille type de la nouvelle bourgeoisie du nord-est de la péninsule, par exemple, considère que l'école ne détermine pas nécessairement le succès dans la vie d'un enfant et que celui-ci doit pouvoir aussi consacrer du 
temps à des activités comme l'anglais, les sports, l'informatique, perçues comme plus modernes ou plus pratiques. Ces familles revendiquent en conséquence plus de temps libre après l'école. Par contre, les familles de classes sociales moins aisées et les femmes qui travaillent continuent souvent de revendiquer une école à temps plein où l'élève puisse faire aussi sa vie d'enfant.

\section{L’approche modernisatrice}

Selon les "modernisateurs", l'école traditionnelle donne trop d'importance aux contenus et aux notions en ne se préoccupant pas assez de l'apprentissage lui-même et des résultats en termes de compétences. Ils pensent qu'il faut diminuer la quantité de connaissances et d'informations transmises et améliorer les techniques didactiques et d'évaluation des acquis. La planification des activités est au centre de cette approche : elle est suggérée par les manuels scolaires qui proposent des unités didactiques contenant des informations bien sélectionnées et des exercices destinés à permettre de mieux les acquérir.

Ces manuels, dont la structure est centrée sur l'apprentissage de l'élève, diffèrent aussi des ouvrages traditionnels en ce que la marge d'autonomie et d'initiative des enseignants, qui n'ont plus la charge de sélectionner eux-mêmes les contenus, y est réduite. Les enseignants apprennent ces méthodes à l'occasion de cours de formation ou simplement en se réunissant à l'école entre collègues autour du manuel en question. Cette approche, qui s'inspire du béhaviorisme nord-américain, est diffusée dans toute l'école obligatoire, dans les écoles à temps plein et dans les autres.

Pour l'élève, le cours magistral se réduit alors à un bref exposé, suivi d'un exercice prescrit par l'enseignant, qui sera corrigé à la fin du cours. Le but des exercices et des tests est de bien fixer les concepts traités. Les outils de travail de l'enfant sont les manuels et les livres d'exercices, des polycopiés et aussi, pour les disciplines scientifiques, des objets et matériaux simples à travailler.

Les contenus étant réduits, le travail en classe devient prépondérant : « on travaille plus en classe qu'à la maison », disent la plupart des enseignants qui utilisent cette approche. Il convient toutefois de noter qu'il arrive souvent que l'on demande à l'élève de l'école Media de réviser chez lui le cours développé en classe ou de compléter un exercice ou un test.

Dans cette approche, l'oral perd de sa suprématie, et on lit moins de textes longs. L'élève est appelé à donner des réponses courtes, l'interrogation traditionnelle à l'estrade n'est plus utilisée.

L'enseignant attache la plus grande importance à la participation de l'élève au travail de la classe et la capacité de ce dernier à suivre le rythme du cours devient un des éléments pris en considération pour l'évaluation. Celle-ci devient plus précise et "objective » que l'évaluation traditionnelle parce qu'elle se fonde surtout sur les résultats obtenus à des tests. Toutefois la tradition de l'évaluation "globale» au sein du conseil de classe n'est pas encore abandonnée. 
Les contenus disciplinaires étant restreints et les exercices étant faits pendant le temps scolaire, le temps de non-école devient plus long et ouvert à toute possibilité d'initiative des familles. L'école fait apprendre le minimum, la famille complète.

\section{L'approche de la transmission des savoirs pour un sujet acteur de son développement}

Il existe en Italie comme dans d'autres pays un important mouvement d'enseignants qui, dans une perspective culturelle et politique, se sont engagés dans une pédagogie qui s’intéresse en même temps à la transmission des savoirs et aux modalités de participation active des élèves. Cette approche diffère de la précédente parce qu'elle donne son importance aux idées de "goût ", de " plaisir de connaître » et dépasse la conception traditionnelle de la culture par l'idée d'implication active de l'élève. L'idée dominante, en rupture avec l'approche traditionnelle, est que la culture doit appartenir et être diffusée à tous.

Ce courant se distingue de l'approche "modernisatrice» par une conception de l'élève (et du citoyen) comme sujet complexe (sujet de connaissance, d'affectivité, d'expériences de vie) et par l'importance donnée aux contenus et connaissances transmises. Si on peut trouver cette approche dans tout type d'école, les enseignants qui s'y réfèrent préfèrent toutefois travailler dans une école à temps plein ou à temps prolongé.

Dans cette approche, les possibilités de mise au travail des élèves sont variées, mais toutes cherchent à faire du travail scolaire un "moment de vie ». En classe on travaille avec l'enseignant, en groupe ou individuellement et les textes proposés sont à dessein divers. Dans les disciplines scientifiques, ce sont l'observation, l'expérimentation, la reconstruction des expériences au laboratoire qui dominent. Dans le domaine linguistique, la communication se développe par tous les moyens et se nourrit souvent des contenus d'expérience de vie personnelle.

Dans cette perspective certaines traditions de l'école italienne sont revisitées. À la différence d'autres pays où l'élève est invité à "l'objectivation » de sa pensée, et à ne pas s'exprimer à la première personne, le tema est traditionnellement en Italie un travail écrit qui constitue un espace pour exprimer des opinions, de sentiments et des pensées. S'il est vrai qu'au cours des années 70-80 cet espace était devenu moins important, les enseignants l'ont récemment redécouvert et, à côté du résumé et du commentaire de texte, nous retrouvons le tema, c'est à dire le développement libre d'un sujet.

Récemment aussi, l'oral qui avait été mis d'un côté, reprend de nouveau une place importante. "Les jeunes de nos jours ne sont plus habitués à la parole - constatent les enseignants - l'école doit modifier cette attitude ». L'exposé oral retrouve son importance même dans les disciplines scientifiques où les enseignants considèrent capital que l'élève reconstruise oralement les 
expériences faites au laboratoire ou qu'il sache exposer une démonstration en mathématiques. Cette signification de l'oral est très différente de celle qu'il avait dans l'école traditionnelle: ici l'oral a comme but la communication de connaissances construites, non l'initiation au discours rhétorique.

Les enseignants qui suivent l'approche ici considérée pensent que le travail à la maison "c'est la réflexion dans la vie »: il faut donc travailler à la maison non pour compléter le programme, mais pour approfondir, pour mieux comprendre et apprendre ce que l'on étudie en classe. Chez lui, l'élève doit lire des textes plus longs, des romans; en sciences, il doit réfléchir aux expériences faites au laboratoire ou aux observations faites au cours d'une sortie, il doit aussi faire de petites recherches.

Les contrôles sont de plusieurs types: au quotidien sur de brèves parties du programme et, sous forme d'interrogations orales ou écrites plus approfondies, à une date fixée longtemps à l'avance. Des contacts continus avec les familles sont nécessaires pour qu'à la maison les devoirs soient faits. Les familles répondent inégalement à cette attente, ce qui repose la question de l'environnement d'origine comme au temps de Don Milani.

Les approches que nous venons de décrire sont des «idéaux-types ». En tant que telles, elles existent rarement dans leur expression "pure ». Malgré la polémique entre les supporters de l'une ou de l'autre manière de travailler à l'école, les approches se mélangent souvent en fait et l'une emprunte aux autres des solutions qui semblent mieux fonctionner avec les élèves; souvent, dans la même classe, les élèves peuvent être exposés à différentes approches et donc à différents types de travail, ce qui, à mon avis, constitue une richesse pour l'élève et permet au surplus un dialogue entre les enseignants et les familles.

Ce qu'on peut se demander dans le contexte actuel de l'application de la loi sur l'Autonomie des établissements, c'est si la diversité des approches sera utilisée en tant que richesse pour améliorer le travail de l'enfant et son apprentissage ou bien pour accentuer la compétition entre les établissements et séparer les enfants, selon l'approche que les familles privilégient.

\section{BIBLIOGRAPHIE}

MILANI D. (1967) : Scuola di Barbiana, lettera ad una professoressa, Editrice fiorentina.

EURYDICE (1999): Les chiffres clés de l'éducation, Bruxelles.

RAGAZZINI D. (1997) : Tempi di scuola, tempi di vita, Nuova Italia.

MINISTERIO DELLA PUBBLICA ISTRUZIONE : Sedi, classi, dotazioni organiche del personale docente della scuola statale 2001/2002, Roma. www.istruzione.it/pubblicazioni 2001. 